\title{
A proportional approach to claims problems with a guaranteed minimum.
}

\author{
José M. Jiménez-Gómez ${ }^{\mathrm{a}}$, Josep E. Peris ${ }^{\mathrm{b}}$ \\ ${ }^{a}$ Universitat Rovira $i$ Virgili, Dep. d'Economia, CREIP and GRODE, \\ Av.Universitat 1, 43204 Reus, Spain. \\ (e-mail: josemanuel.jimenez@urv.cat) \\ ${ }^{b}$ Universitat d'Alacant, Dep. de Mètodes Quantitatius $i$ Teoria Econòmica, \\ 03080 Alacant, Spain. \\ (e-mail: peris@ua.es)
}

\begin{abstract}
In distribution problems, and specifically in bankruptcy issues, the Proportional $(P)$ and the Egalitarian $(E A)$ divisions are two of the most popular ways to resolve the conflict. Nonetheless, when using the egalitarian division, agents may receive more than her claim. We propose a compromise between the proportional and the egalitarian solutions by considering the restriction that no one receives more than her claim. We show that the most egalitarian compromise fulfilling this restriction ensures a minimum amount to each agent, and it can be interpreted as a process that works in two steps as follows: first, all agents receive an equal share up to the smallest claim (egalitarian distribution), and then the remaining estate (if any) is allocated proportionally to the remaining claims (proportional solution). Finally, we obtain that the recursive application of this process finishes at the Constrained Equal Awards solution (CEA).
\end{abstract}

Keywords: Claims problems, Proportional solution, Equal Awards, Compromise, Lorenz dominance JEL classification: C71, D63, D71.

\section{Introduction.}

A claims problem is a particular case of distribution problems, in which the amount to be distributed, the estate $E$, is not enough to cover the agents' claims on it. This model describes the situation faced by a court that has to 
distribute the net worth of a bankrupt firm among its creditors, but it also corresponds with cost-sharing, taxation, or rationing problems. How should the scarce resources be allocated among its claimants? The formal analysis of situations like these, which originates in a seminal paper by O'Neill (1982), shows that a vast number of well-behaved solutions have been defined for solving claims problems, being the Proportional and the Equal Awards (egalitarian) the two prominent concepts used in real world. ${ }^{1}$ The term wellbehaved reflects the idea that the considered solutions might fulfill some principles of fairness, or appealing properties. Furthermore, an equity principle that has been used to compare solutions is that of Lorenz-dominance (see Dutta and Ray (1989)). A recent paper (Bosmans and Lauwers (2011)) compares the most usual bankruptcy rules in terms of Lorenz-dominance and analyzes those solutions that favour to smaller claimants relative to larger ones.

Although the Proportional division is the most used, whenever the smallest claim is very small compared with the largest one, a proportional division provides nearly nothing for this (these) small claimant(s). ${ }^{2}$

An illustrative example of claims problems is the fishing quotas reduction, in which the agent's claim can be understood as the previous captures, and the estate is the new (lower) level of joint captures. A similar example is given by milk quotas among the EU members. ${ }^{3}$ In both examples, proportionality is the main principle used. Nevertheless, a minimal (survival) amount, guaranteed to each producer, should be fixed in order to ensure the profitability of fishing (milk) industries. That is, some part of the estate should be allocated in an egalitarian way. This idea is somewhat related to the axiom of Sustainability (see Herrero and Villar (2002)). As they mention,

"Sustainability is a protective criterion for those agents with small claims. To illustrate this, consider the interpretation of a bankruptcy situation as a reduction in the fishing quotas. Here agent i's claim corresponds to her actual level of captures and

\footnotetext{
${ }^{1}$ The reader is referred to the survey by Thomson (2003).

2 "In western society, for example, the customary solution would be to split the asset in proportion topg. the claims", see Young (1994), pg. 123.

${ }^{3}$ Quotas were introduced in 1984 . Each member state was given a reference quantity which was then allocated to individual producers. The initial quotas were not sufficiently restrictive as to remedy the surplus situation and so the quotas were cut in the late 1980s and early 1990s. Quotas will end on April 1, 2015.
} 
the estate to be distributed to the new aggregate level of captures. Sustainable claims correspond to those levels of captures such that, if nobody else had a larger level, the aggregate new level of captures would not impose any rationing. Sustainability says that agents with sustainable claims should not be rationed after the change in the aggregate level of captures."

A similar situation can be found when a university distributes the budget to Departments. In this case, the resources are distributed proportionally to the number of Professors, students, subjects, etc., but a minimal (fixed) amount is allocated to each regardless of size.

An alternative example of using the proportional approach is the way in which seats in the Spanish Parliament are allocated to each of the electoral districts (provinces). ${ }^{4}$ This is made proportionally to the population in each province, but a minimal number of seats (2) is guaranteed to each. ${ }^{5}$ A similar situation is found in the US case: based on data from the decennial census, each state is allocated a proportion of the 435 seats in the United States House of Representatives, although each state is guaranteed a minimum of one seat, regardless of population. ${ }^{6}$ The remaining seats are allocated one at a time, to the state with the highest priority number. This apportionment is based on the proportion of each state's population to that of the Fifty States together. We shall return to these examples later.

The previous comments and examples show that real world, when applying proportional distributions, try to ensure an egalitarian (minimal) amount

\footnotetext{
${ }^{4}$ This example involves indivisibilities, which is not a trivial issue (see, for instance, Herrero and Martínez (2004)).

${ }^{5}$ In the case of Spanish Parliament, the allocation mechanism is as follows (Spanish LOREG, 2011, art. 162): [1.] Congress is composed of three hundred and fifty Deputies. [2.] Each province has a corresponding initial minimum of two deputies. [3.] The remaining two hundred and forty-eight deputies are distributed among the provinces in proportion to its population, according to the following procedure: a) Obtain a distribution fee obtained by dividing by two hundred forty-eight the total number of the legal population of peninsular and island provinces. b) Allocate to each province as many deputies as resulting, in whole numbers, dividing the population of provincial law by the quota allocation. c) The remaining deputies are distributed by assigning one to each of the provinces whose quotient obtained under paragraph before, have a higher decimal fraction.

6 "Each State shall have at Least one Representative" (U.S. Const., art. I, 2, cl. 3.).
} 
to each agent. In this paper we will define a new solution concept that captures this behavior. This solution can be understood as a compromise between the proportional and the egalitarian division. Noting that the first (proportional) distribution is a widely used solution for claims problems, and that the second distribution (equal awards, which is not a solution since an agent may receive an award larger than her claim) is the most egalitarian distribution available, we search for a compromise between them. In choosing this compromise, if for each problem we wanted to use the same weight on the proportional and the egalitarian distributions, the largest weight one could assign to the egalitarian distribution would be zero (otherwise for some problems an agent would receive an amount larger than her claim). So, we propose that the weight of each of the two distributions depends on each claims problem. In so doing, we define the weight used on the egalitarian distribution to be the highest weight such that resulting vector satisfies the claims boundedness restriction.

On the other hand, we may differentiate between two different kinds of problems: the first kind are problems where the per-capita estate is small relative to the smallest claim, $c_{1} \geq E$ (a condition called in the literature as an unsustainable claim), and the second where the smallest claim is sustainable. Then, if the claims problem is in the first category, the egalitarian distribution satisfies claims boundedness and all agents receive equal awards; if the claims problem falls in the second category, we first assign to each agent the smallest claim (egalitarian distribution), revise claims and estate accordingly, and then distribute the remaining estate proportionally to the revised claims (proportional solution). By this way, we define a new solution. Our main result, Proposition 3, shows that both approaches coincide in the same solution which we call $\alpha_{m i n}-$ Egalitarian solution. ${ }^{7}$

In short, our compromise solution:

- modifies the Equal Awards division, so that the proposal satisfies the claim-boundedness condition and it is a bankruptcy solution;

- modifies the Proportional solution and considers a minimal amount

\footnotetext{
${ }^{7}$ An interesting question that has been addressed to us is if we can do the same for any bankruptcy solution $\psi$ instead of the Proportional one. We will see that it is not possible, in general, to extend our results.
} 
that each agent should receive. ${ }^{8}$ This minimal amount is endogenously determined in each particular problem $(E, c)$;

- the result it provides is the same that we will obtain if we assign to each agent this minimal amount, and distribute the remaining (if there exist) in a proportional way.

The paper is organized as follows: Section 2 contains the preliminaries. Section 3 presents our solution concept. Sections 4 and 5 contain the axiomatic analysis. Finally, Section 6 contains some comments. The Appendix gathers the proofs.

\section{Preliminaries: claims problems.}

Throughout the paper we will consider a set of agents $N=\{1,2, \ldots, n\}$. Each agent is identified by her claim, $c_{i}, i \in N$, on the estate E. A claims problem appears whenever the estate is not enough to satisfy all the claims; that is, $\sum_{i=1}^{n} c_{i}>E$. Without loss of generality, we will order the agents according to their claims: $c_{1} \leq c_{2} \leq \cdots \leq c_{n}$. The pair $(E, c)$ represents the claims problem, and we will denote by $\mathcal{B}$ the set of all claims problems. A claims solution (solution) is a single valued function $\varphi: \mathcal{B} \rightarrow \mathbb{R}_{+}^{n}$ such that, $0 \leq \varphi_{i}(E, c) \leq c_{i}, \forall i \in N$ (non-negativity and claim-boundedness), and $\sum_{i=1}^{n} \varphi_{i}(E, c)=E($ efficiency $)$

Many solution concepts have been defined in the literature about claims problems (see for instance Thomson (2003), and Bosmans and Lauwers (2011)). The two most important criteria are the Proportional and the Egalitarian ones.

Definition 1. The Proportional solution, P. For each $(E, c) \in \mathcal{B}$ and each $i \in N, P_{i}(E, c)=\lambda c_{i}$, where $\lambda=\frac{E}{\sum_{i \in N} c_{i}}$.

\footnotetext{
${ }^{8}$ Our proposal satisfies a lower bound on awards property; see Section 4.
} 
Definition 2. The Equal Awards division, EA. For each $(E, c) \in \mathcal{B}$ and each $i \in N, E A_{i}(E, c)=\frac{E}{n}$.

It is easy to find examples in which the equal distribution of the estate exceeds some agent's claim. ${ }^{9}$ So that, the $E A$ division is not a solution, in the sense we have defined it ( $E A$ may not satisfy the second part of the first condition of a solution: claim-boundedness). In order to solve this situation the following modification of the $E A$ division has been introduced.

Definition 3. The Constrained Equal Awards solution, CEA. For each $(E, c) \in \mathcal{B}$ and each $i \in N, C E A_{i}(E, c) \equiv \min \left\{c_{i}, \mu\right\}$, where $\mu$ is chosen so that $\sum_{i \in N} \min \left\{c_{i}, \mu\right\}=E$.

\section{A proposal of solution: $\alpha_{\text {min }}$-Egalitarian}

Given the Proportional and the Egalitarian divisions, we consider now the family of compromises:

$$
\varphi_{\alpha}=\alpha P+(1-\alpha) E A \quad \alpha \in[0,1] .
$$

That is, given a claims problem $(E, c)$ involving $n$ agents,

$$
\left(\varphi_{\alpha}\right)_{i}(E, c)=\alpha \frac{c_{i} E}{\sum_{i=1}^{n} c_{i}}+(1-\alpha) \frac{E}{n} \quad \alpha \in[0,1] .
$$

The following example computes this proposal for several values of $\alpha$.

Example 1. Consider $(E, c)=(100,(40,50,70))$.

\begin{tabular}{|c|c|c|c|c|c|}
\hline Claims & $\alpha=0$ & $\alpha=0.25$ & $\alpha=0.50$ & $\alpha=0.75$ & $\alpha=1$ \\
\hline 40 & $100 / 3$ & 31.25 & 29.17 & 27.08 & 25 \\
\hline 50 & $100 / 3$ & 32.81 & 32.29 & 31.77 & 31.25 \\
\hline 70 & $100 / 3$ & 35.94 & 38.54 & 41.15 & 43.75 \\
\hline
\end{tabular}

\footnotetext{
${ }^{9}$ For instance, consider the claims vector $c=(20,50,60)$ and the estate $E=100$.
} 
As we have already mentioned, when $\alpha=0$ the equal division may not satisfy the conditions of a solution (claim boundedness fails). In order to avoid this problem, we can obtain for every problem $(E, c)$ the minimum value of $\alpha \in[0,1]$ such that $\varphi_{\alpha}$ is a solution:

$$
\alpha^{*}(E, c)=\min \left\{\alpha \in[0,1] \text { such that }\left(\varphi_{\alpha}(E, c)\right)_{i} \leq c_{i} \quad \forall i\right\} .
$$

Remark 1. Note that if we solve, for each agent $i$, the equation

$$
\alpha_{i}: \quad\left(\varphi_{\alpha}(E, c)\right)_{i}=c_{i},
$$

then

$$
\alpha^{*}(E, c)=\max \left\{\alpha_{1}, \alpha_{2}, \ldots, \alpha_{n}\right\}
$$

Definition 4. The $\alpha_{\min }-$ Egalitarian solution is defined for every claims problem $(E, c)$, with $c_{i}>0 \quad \forall i \in N$, as:

$$
\varphi_{\min }(E, c)=\varphi_{\alpha^{*}}(E, c)
$$

where $\alpha^{*}=\alpha^{*}(E, c)$

Note that $\alpha^{*}$ varies from a claims problem to another. However, by the way it is defined, the $\alpha_{\min }-$ Egalitarian solution is continuous. Next, we consider a consistent extension of our solution in the presence of null claims, and we analyze the way of obtaining $\alpha^{*}(E, c)$.

Definition 5. If there are some zero claims, $c_{1}=c_{2}=\ldots=c_{k}=0, c_{j}>0$, for all $j>k$, we extend our solution in a consistent way:

$$
\varphi_{\min }(E, c)=\left(\boldsymbol{O}, \varphi_{\min }(E, \bar{c})\right) \quad \boldsymbol{O}=(0, \ldots, 0)_{1 \times k} \quad \bar{c}=\left(c_{k+1}, \ldots, c_{n}\right)
$$

Proposition 1. If the claim boundedness is fulfilled by the agent with lowest claim, it is fulfilled by any agent:

$$
\left(\varphi_{\alpha}(E, c)\right)_{1} \leq c_{1} \Rightarrow\left(\varphi_{\alpha}(E, c)\right)_{i} \leq c_{i} \quad \forall i .
$$

See the proof in the Appendix. 
Remark 2. The result in the above proposition does not remain true if we use, in order to define $\varphi_{\alpha}$, a solution $\psi$ different from the Proportional one

$$
\varphi_{\alpha}=\alpha \psi+(1-\alpha) E A \quad \alpha \in[0,1] .
$$

For instance, if we consider the problem $(E, c)=(90,(10,12,100))$ and a solution $\psi$ such that $\psi(90,(10,12,100))=(8,11,71)$, then the second agent is the one who defines $\alpha^{*}(E, c)=\frac{18}{19}$. See Section 6 .

In the following result we show the exact expression of $\alpha^{*}$.

Proposition 2. Given a claims problem $(E, c)$ the scalar $\alpha^{*}$ is:

$$
\alpha^{*}(E, c)=\max \left\{0, \frac{C\left(E-n c_{1}\right)}{E\left(C-n c_{1}\right)}\right\} \quad C=\sum_{i=1}^{n} c_{i}
$$

See the proof in the Appendix.

Remark 3. From this expression, it is immediate to see that, for $E \leq n c_{1}$, $\alpha^{*}(E, c)=0$ and, for $E \geq n c_{1}, \alpha^{*}(E, c)$ is an strictly increasing and concave function of $E$ for fixed claims vector $c$, as shown in Figure 1.

Now, trying to facilitate the comparison with the main solutions in the literature, we compute our proposal for the next two examples taken from Bosmans and Lauwers (2011). ${ }^{10}$

Example 2. $(E, c)=(1500,(500,2000,3500))$.

\begin{tabular}{|c|c|c|c|c|c|c|}
\hline$c_{i}$ & $C E A, \varphi_{\min }$ & Pin, T, CE & $A$ & $R A, M O$ & $P$ & $C E L$ \\
\hline 500 & 500 & 250 & 214 & 166.7 & 125 & 0 \\
\hline 2000 & 500 & 625 & 643 & 666.7 & 500 & 0 \\
\hline 3500 & 500 & 625 & 643 & 666.7 & 875 & 1500 \\
\hline
\end{tabular}

with $\alpha^{*}(E, c)=0$.

\footnotetext{
${ }^{10}$ Hereinafter, Pin, T, CE, A, RA, MO, and CEL will denote the Piniles', Talmud, Constrained Egalitarian, Adjusted Proportional, Random Arrival, Minimal Overlap and Constrained Equal Losses solutions, respectively. See Thomson (2003) for their formal definitions.
} 


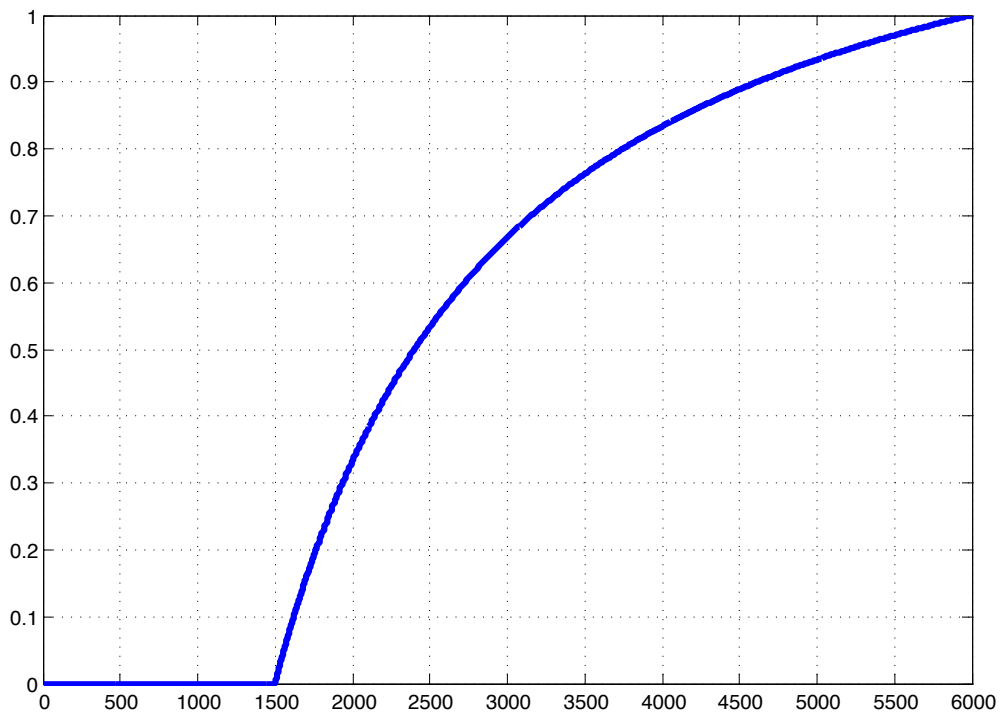

Figure 1: $\alpha^{*}(E, c)$ as a function of $E$ for fixed claims $(c=(500,2000,3500))$.

Example 3. $(E, c)=(4500,(500,2000,3500))$.

\begin{tabular}{|c|c|c|c|c|c|c|c|c|c|}
\hline$c_{i}$ & $C E A, C E$ & $P$ in & $\varphi_{\min }$ & $P$ & $R A$ & $A$ & $T$ & $M O$ & $C E L$ \\
\hline 500 & 500 & 500 & 500 & 375 & 333.3 & 285.72 & 250 & 166.7 & 0 \\
\hline 2000 & 2000 & 1625 & 1500 & 1500 & 1333.3 & 1357.14 & 1375 & 1416.7 & 1500 \\
\hline 3500 & 2000 & 2375 & 2500 & 2625 & 2333.3 & 2857.14 & 2875 & 2916.7 & 3000 \\
\hline
\end{tabular}

with $\alpha^{*}(E, c)=\frac{8}{9}$.

Finally, in the following result, we find a precise expression of our solution which gives us an interesting interpretation: this solution assigns the minimal claim to any agent; thus it distributes the remaining estate $E^{\prime}=E-n c_{1}$ in a proportional way among the agents with respect to the remaining claims $c_{i}^{\prime}=c_{i}-c_{1}$. The proof is given in the Appendix. 
Proposition 3. For each $(E, c) \in \mathcal{B}$, with $c_{i}>0$, for all $i$,

$$
\begin{aligned}
& \varphi_{\min }(E, c)= \begin{cases}(E / n) \mathbf{1} & c_{1} \geq E / n \\
\boldsymbol{c}^{1}+P\left(E-n c_{1}, c-\boldsymbol{c}^{1}\right) & \text { otherwise }\end{cases} \\
& \text { where } \boldsymbol{c}^{1}=\left(\begin{array}{c}
c_{1} \\
\ldots \\
c_{1}
\end{array}\right)_{n \times 1} \text { and } \mathbf{1}=\left(\begin{array}{c}
1 \\
\ldots \\
1
\end{array}\right)_{n \times 1}
\end{aligned}
$$

The condition that splits both cases in Proposition 3 is known in the literature with the name of sustainable claim. ${ }^{11}$ Note that if the smaller claim $c_{1}$ is not sustainable, $c_{1}>E / n$, then no claim is sustainable. Therefore, the result in Proposition 3 can be stated as:

- If $c_{1}$ is sustainable, then $\varphi_{\min }(E, c)=\mathbf{c}^{1}+P\left(E-n c_{1}, c-\mathbf{c}^{1}\right)$.

- If $c_{1}$ is not sustainable, then $\varphi_{\min }(E, c)=E A(E, c)$.

In Figure 2 we represent the distribution of the estate, by depending on $E$, given by the $\alpha_{\text {min }}-$ Egalitarian solution.

Remark 4. It is important to mention that, as in Remark 2, the result in Proposition 3 is not true if we use a solution $\psi$ different from the Proportional one. See Section 6.

\footnotetext{
${ }^{11} \mathrm{~A}$ claim $c_{i}$ is said to be sustainable in $(E, c)$, (see Herrero and Villar (2002)) if $\sum_{j=1}^{n} \min \left\{c_{i}, c_{j}\right\} \leq E$
} 


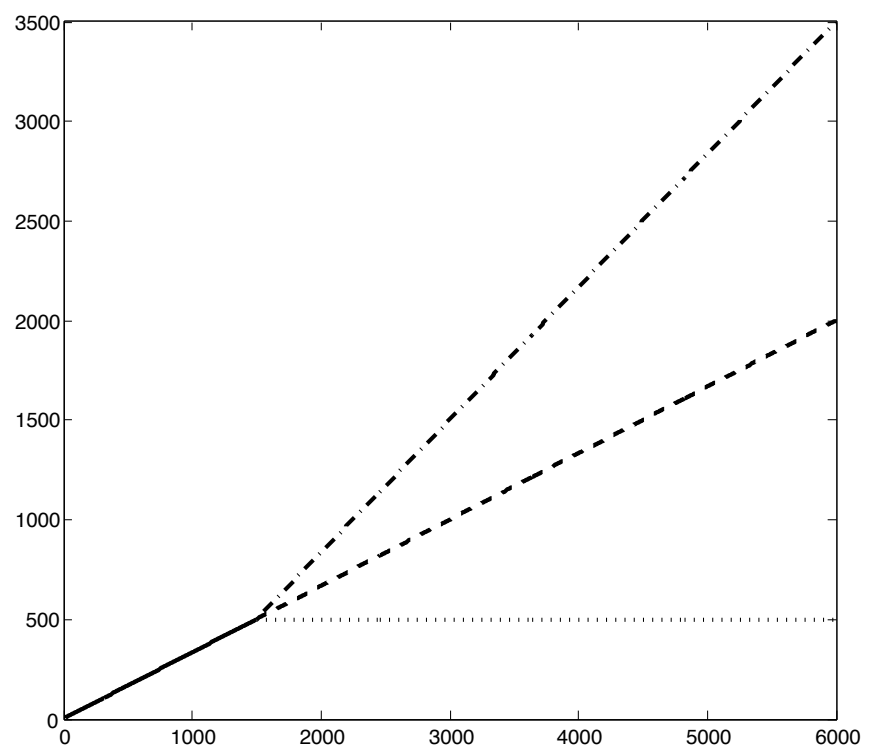

Figure 2: The $\alpha_{m i n}-$ Egalitarian solution. The horizontal axis represents different levels of the estate $E$, and vertical axis denotes the amount each agent receives according their claims, $c=(500,2000,3500)$. The solid black line represents the egalitarian distribution of the estate our proposal obtains when $E \leq 1500$. From this point on, our proposal recommends the pointed-dashed lines for agents $1,2,3$, from bottom to top, respectively.

\section{Axiomatic analysis and comparison with other solutions.}

In this section we analyze our solution from an axiomatic point of view. First, next table summarizes the axiomatic comparative between the $\alpha_{\text {min }}-$ Egalitarian solution and the ones more directly related to it, $C E A$ and $P$. 


\begin{tabular}{lccc}
\hline & $\varphi_{\min }$ & $P$ & $C E A$ \\
\hline Order preservation & Yes & Yes & Yes \\
\hline Resource monotonicity & Yes & Yes & Yes \\
\hline Super-modularity & Yes & Yes & Yes \\
\hline Order preservation under claims variations & Yes & Yes & Yes \\
\hline Composition up & Yes & Yes & Yes \\
\hline Composition down & Yes & Yes & Yes \\
\hline Invariance under claims truncation & No & No & Yes \\
\hline Self-duality & No & Yes & No \\
\hline Midpoint property & No & Yes & No \\
\hline Limited consistency & Yes & Yes & Yes \\
\hline Reasonable lower bounds on awards & Yes & No & Yes \\
\hline
\end{tabular}

In order to check that the $\alpha_{\text {min }}$-Egalitarian solution satisfies, or not, these properties, we formally give their definitions.

Order preservation (Aumann and Maschler (1985)) requires respecting the ordering of the claims: if agent $i^{\prime} s$ claim is at least as large as agent $j^{\prime} s$ claim, she should receive and lose at least as much as agent $j$ does, respectively.

Order preservation: for each $(E, c) \in \mathcal{B}$, and each $i, j \in N$, such that $c_{i} \geq c_{j}$, then $\varphi_{i}(E, c) \geq \varphi_{j}(E, c)$, and $c_{i}-\varphi_{i}(E, c) \geq$ $c_{j}-\varphi_{j}(E, c)$.

Resource monotonicity (Curiel et al. (1987), Young (1987)) demands that if the endowment increases, then all individuals should get at least what they received initially.

Resource monotonicity: for each $(E, c) \in \mathcal{B}$ and each $E^{\prime} \in \mathbb{R}_{+}$ such that $C>E^{\prime}>E$, then $\varphi_{i}\left(E^{\prime}, c\right) \geq \varphi_{i}(E, c)$, for each $i \in N$.

Super-modularity (Dagan et al. (1997)) requires that if the amount to divide increases, given two individuals, the one with the greater claim experiences a larger gain than the other.

Super-modularity: for each $(E, c) \in \mathcal{B}$, all $E^{\prime} \in \mathbb{R}_{+}$and each $i, j \in N$ such that $C>E^{\prime}>E$ and $c_{i} \geq c_{j}$, then $\varphi_{i}\left(E^{\prime}, c\right)-$ $\varphi_{i}(E, c) \geq \varphi_{j}\left(E^{\prime}, c\right)-\varphi_{j}(E, c)$. 
Reasonable lower bounds on awards (Moreno-Ternero and Villar (2004); Dominguez and Thomson (2006)) ensures that each individual receives at least the minimum of (i) her claim divided by the number of individuals, and (ii) the amount available divided by the number of individuals.

Reasonable lower bounds on awards: for each $(E, c) \in \mathcal{B}$ and each $i \in N, \varphi_{i}(E, c) \geq \frac{\min \left\{c_{i}, E\right\}}{n}$.

Order preservation under claims variations (Thomson (2006)) requires that if the claim of some individual decreases, given two other individuals, the one with the greater claim experiences a larger gain than the other.

Order preservation under claims variations: for each $k \in$ $N$, each pair $(E, c)$ and $\left(E, c^{\prime}\right) \in \mathcal{B}$, with $c^{\prime}=\left(c_{k}^{\prime}, c_{-k}\right)$ and $c_{k}^{\prime}<c_{k}$ and each pair $i$ and $j \in N \backslash k$ with $c_{i} \leq c_{j}, \varphi_{i}\left(E, c^{\prime}\right)-\varphi_{i}(E, c) \leq$ $\varphi_{j}\left(E, c^{\prime}\right)-\varphi_{j}(E, c) .{ }^{12}$

Composition down requires that if, after the resources are distributed, they are reduced, a solution recommends the same allocation if we (i) cancel the initial distribution and apply the solution in the new situation, or (ii) consider the initial awards as agents' claims on the revised problem and apply the solution to this new problem.

Composition down: for each $(E, c) \in B$, each $i \in N$, and each $0 \leq E^{\prime} \leq E, \varphi_{i}\left(E^{\prime}, c\right)=\varphi_{i}\left(E^{\prime}, \varphi(E, c)\right)$.

Composition up shows the opposite situation to composition down. If, after the resources are distributed, they are increased, a solution recommends the same allocation if we (i) cancel the initial distribution and apply the solution in the new situation, or (ii) let agents keep their initial awards, adjust claims down by these amounts, and reapply the solution to divide only the increment of the estate with these adjusted claims.

Composition up: for each $\left(E^{\prime}, c\right) \in B$, each $i \in N$, and each $0 \leq E \leq E^{\prime}, \varphi_{i}\left(E^{\prime}, c\right)=\varphi_{i}(E, c)+\varphi_{i}\left(E^{\prime}-E, c-\varphi(E, c)\right)$.

\footnotetext{
${ }^{12}$ We write $\left(c_{k}^{\prime}, c_{-k}\right)$ for the claims vector obtained from $c$ by replacing $c_{k}$ by $c_{k}^{\prime}$.
} 
Limited consistency states that adding an agent with a zero claim does not change the awards of the individuals already present. Obviously, if $\left(E,\left(c_{1}, c_{2}, \ldots, c_{n}\right)\right)$ is a claims problem involving $n$ individuals, then $\left(E,\left(0, c_{1}, c_{2}, \ldots, c_{n}\right)\right)$ is a problem with $n+1$ individuals.

Limited consistency: for each $(E, c) \in \mathcal{B}$, for all $i=1,2, \ldots, n$ $\varphi_{i}(E, c)=\varphi_{i}\left(E,\left(0, c_{1}, \ldots, c_{n}\right)\right)$.

Next Proposition, whose proof is given in the Appendix, shows that the $\alpha_{\min }-$ Egalitarian solution fulfills the above mentioned properties.

Proposition 4. The $\alpha_{m i n}-$ Egalitarian solution fulfills Order preservation, Resource monotonicity, Super-modularity, Reasonable lower bounds on awards, Order preservation under claims variations, Composition up, Composition down and Limited consistency.

Remark 5. Note that there is a property our solution fulfills that is not satisfied by the Proportional solution: Reasonable lower bounds on awards. This is the part that the EA division brings to our solution. The drawback is that some properties $P$ fulfills are lost. Next we show some of them. ${ }^{13}$

Self-Duality implies that a solution recommends the same allocation when dividing awards and losses. Given a claims problem $(E, c)$, losses are defined by the difference among the estate and the claims, $L=\sum_{i \in N} c_{i}-E$.

Self-duality: for each $(E, c) \in \mathcal{B}$ and each $i \in N, \varphi_{i}(E, c)=$ $c_{i}-\varphi_{i}(L, c)$.

Midpoint Property ensures to each agent half of her claim when the estate equals half of the aggregate claim.

Midpoint Property: for each $(E, c) \in \mathcal{B}$ and each $i \in N$, if $E=C / 2$, then $\varphi_{i}(E, c)=c_{i} / 2$.

\footnotetext{
${ }^{13}$ It must be noticed that the main reason for not satisfying these properties is that $E A$, taken as a function, does not satisfy them.
} 
Invariance under claims truncation tells us that the part of a claim that is above the resources should not be taken into account.

Invariance under claims truncation: for each $(E, c) \in \mathcal{B}$ and each $i \in N, \varphi_{i}(E, c)=\varphi_{i}\left(\left(E, \min \left\{c_{i}, E\right\}_{i \in N}\right)\right.$.

The following example shows that the $\alpha_{\min }-$ Egalitarian solution does not satisfy these properties.

Example 4. Consider $(E, c)=(2000,(500,2000,3500))$. Then

$$
\varphi_{\min }(E, c)=(500,666.66,833.33) .
$$

$(L, c)=(4000,(500,2000,3500))$, and $\varphi_{\min }(L, c)=(500,1333.33,2166.66)$.

So, $c-\varphi_{\min }(L, c)=(0,727.28,1272.73) \neq \varphi_{\min }(E, c)$, not satisfying Selfduality.

Midpoint property implies $\varphi(E, c)=(250,1000,1750) \neq \varphi_{\min }(E, c)$.

For $\left(E, c^{\prime}\right)=(2000,(500,2000,2000)), \alpha_{\min }\left(E, c^{\prime}\right)=(500,750,750) \neq$ $\varphi_{\min }(E, c)$, not satisfying Invariance under claims truncation.

Finally, we introduce an operation for solutions that will help us to analyze the iterative application of the $\alpha_{\min }-$ Egalitarian solution. We name this operation Self-composition, since it is related to the Self-consistency property (see for instance Grahn and Voorneveld (2002)). ${ }^{14}$ In particular, Self-composition proposes a "recursive" distribution of the resources starting from agent 1. Formally,

Definition 6. Self-composition: for each $(E, c) \in \mathcal{B}$, and each $m, 1 \leq$ $m \leq n$, the Self-composition of degree $m$ is defined by:

$\varphi^{m}(E, c)=\left(\varphi_{1}\left(E^{1}, c^{1}\right), \ldots, \varphi_{m-1}\left(E^{m-1}, c^{m-1}\right), \varphi_{m}\left(E^{m}, c^{m}\right), \ldots, \varphi_{n}\left(E^{m}, c^{m}\right)\right)$

where $\left(E^{1}, c^{1}\right)=(E, c)$ and for all $k>1$,

$$
E^{k}=E^{k-1}-\varphi_{k-1}\left(E^{k-1}, c^{k-1}\right) ; \quad c^{k}=\left(0, \ldots, 0, c_{k}, \ldots, c_{n}\right) .
$$

\footnotetext{
${ }^{14}$ Self-consistency: for each $(E, c) \in \mathcal{B}$, each $S \subseteq N$ and each $i \in S$, then $\varphi_{i}(E, c)=$ $\varphi_{i}\left(\sum_{k \in S} \varphi_{k}(E, c), c_{\mid S}\right)$.
} 
For instance, the Self-composition of degree 2 for some solution, $\varphi^{2}$, is obtained in the following way: first, agent 1 receives the amount recommended for her by $\varphi(E, c)$; then we solve the new problem in which the estate is reduced in the amount given to agent 1 , and this agent has no claim anymore. That is, $\varphi^{2}(E, c)=\left(\varphi_{1}(E, c), \varphi_{2}\left(E^{2}, c^{2}\right), \varphi_{3}\left(E^{2}, c^{2}\right), \ldots, \varphi_{n}\left(E^{2}, c^{2}\right)\right)$ where $E^{2}=E-\varphi_{1}(E, c) ; \quad c^{2}=\left(0, c_{2}, \ldots, c_{n}\right)$.

It is immediate to observe that if a solution is Self-consistent, then the Self-composition of any degree coincides with the own function (in some sense, it is idempotent); i.e., if $\varphi$ satisfies Self-consistency, then

$$
\forall(E, c) \in \mathcal{B}, \quad \forall m \quad \varphi^{m}(E, c)=\varphi(E, c) .
$$

Next result, which can be straightforwardly obtained from Proposition 3 , shows that if we compute the Self-composition of degree $n-1$ of the $\alpha_{\text {min }}-$ Egalitarian solution, we obtain the $C E A$ solution.

Proposition 5. The Self-composition of degree $n-1$ of the $\alpha_{\min }-$ Egalitarian solution retrieves the CEA solution, where $n$ is the number of agents.

The result in the above Proposition may be understood as a recursive process (for a solution $\varphi$ ) which can be described as follows. Assume the agents are ordered so that $c_{1} \leq c_{2} \leq \ldots \leq c_{n}$. The solution $\varphi$ applied to the original problem $(E, c)$ only determines the share of agent 1 , who in turn leaves with this share. The estate is reduced accordingly and the updated problem, say $\left(E^{2}, c^{2}\right)$ for agents $2,3, \ldots, n$ is now used only to determine the share of agent 2. Agent 2 then leaves with this share and the estate is again reduced to construct $\left(E^{3}, c^{3}\right)$ for agents $3,4, \ldots, n$. This recursive process is used to determine the share of every agent. The result shows that this recursive process, when applied to $\varphi_{\min }$, produces the $C E A$ allocation.

The $\alpha_{m i n}-$ Egalitarian solution does not satisfy self-consistency (otherwise, self-composition could not retrieve the $C E A$ solution). But it satisfies a weaker version that we call backwards consistency. This condition requires that if the agent with largest claim leaves with his part, none of the other agents takes advantage.

Definition 7. Backwards Consistency: for each $(E, c) \in \mathcal{B}$,

$$
\varphi(E, c)=\left(\varphi\left(E-\varphi_{n}(E, c),\left(c_{1}, c_{2}, \ldots, c_{n-1}, 0\right)\right), \varphi_{n}(E, c)\right)
$$


It is obvious that Self-consistency implies Backwards-consistency, but the converse is not true as shows the following result in which we prove that the $\alpha_{\text {min }}-$ Egalitarian solution satisfies this property. The proof is given in the Appendix.

Proposition 6. The $\alpha_{\text {min }}-$ Egalitarian solution satisfies Backwards-consistency.

\section{Lorenz dominance.}

An interesting tool to compare the behavior of solution concepts is that of Lorenz dominance. Let $\mathbb{R}_{+}^{n}$ be the set of positive $n$-dimensional vectors $x=\left(x_{1}, x_{2}, \ldots, x_{n}\right)$ ordered from small to large, i.e., $0<x_{1} \leq x_{2} \leq \ldots \leq x_{n}$. Let $x$ and $y$ be in $\mathbb{R}_{+}^{n}$. We say that $x$ Lorenz dominates $y, x \succ_{L} y$, if for each $k=1,2, \ldots, n-1: x_{1}+x_{2}+\cdots+x_{k} \geq y_{1}+y_{2}+\ldots+y_{k}$ and $x_{1}+x_{2}+\ldots+x_{n}=y_{1}+y_{2}+\ldots+y_{n}$. If $x$ Lorenz dominates $y$ and $x \neq y$, then at least one of these $n-1$ inequalities is a strict inequality. The following definition extends the notion of Lorenz dominance to bankruptcy solutions.

Definition 8. Given two solutions $\varphi$ and $\psi$ it is said that $\varphi$ Lorenz dominates $\psi, \varphi \succ_{L} \psi$, if for any claims problem $(E, c)$ the vector $\varphi(E, c)$ Lorenz dominates $\psi(E, c)$.

Lorenz domination is a criterion used to check whether a solution is more favorable to smaller claimants relative to larger claimants. So, in some sense, a Lorenz dominant solution can be understood as more equitable. In a recent paper, Bosmans and Lauwers (2011) obtain a Lorenz dominance comparison among several solutions and they obtain that $C E A$ is the more equitable solution, in the sense that it Lorenz dominates any other solution. More precisely, the dominance relation they obtain is as follows:

$$
C E A \succ_{L} C E \succ_{L} \operatorname{Pin} \succ_{L} P \succ_{L} C E L
$$

Then, the Proportional solution only dominates $C E L$, which is the most favorable solution for larger claimants relative to smaller ones (so, the less equitable one). ${ }^{15}$

\footnotetext{
${ }^{15}$ See Bosmans and Lauwers (2011) for additional relationships.
} 
Among the solutions analyzed in Bosmans and Lauwers (2011), only $C E A$ dominates the $\alpha_{m i n}-$ Egalitarian solution. Next result shows the Lorenz relationships between our solution and the ones on that paper.

\section{Proposition 7.}

a) The $\alpha_{\text {min }}-$ Egalitarian solution Lorenz dominates $P$ and CEL.

b) There is no Lorenz domination between the $\alpha_{\text {min }}-$ Egalitarian solution and $C E$, Pin, RA, MO, T, and A solutions.

Part b), with respect to $C E$ and Pin is directly obtained from examples 2 and 3. Moreover, example 3 shows a claims problem in which the $\alpha_{\text {min }}$-Egalitarian solution Lorenz dominates $R A, M O, T$ and $A$. Next example shows a case in which these solutions are not Lorenz dominated by the $\alpha_{\min }-$ Egalitarian solution.

Example 5. Let $(E, c)=(20,(2,20,40))$. Then,

\begin{tabular}{|c|c|c|c|c|}
\hline$c_{i}$ & $\varphi_{\min }$ & $R A=M O$ & $A$ & $T$ \\
\hline 2 & 2 & 0.66 & 0.96 & 1.9 \\
\hline 20 & 6.5 & 9.66 & 9.52 & 9.5 \\
\hline 40 & 11.5 & 9.66 & 9.52 & 9.5 \\
\hline
\end{tabular}

Proof of part a) is given in the Appendix.

\section{Final comments.}

In this paper we have proposed a compromise between the two most important and well-known ways of solving distribution problems: the Proportional and the Egalitarian. Moreover, we have analyzed the properties of this new solution and defined a recursive process, Self-composition, which allows us to recover the Constrained Equal Awards solution, by using our solution.

A natural question arises at this point: if we consider an alternative solution concept (e.g. Talmud solution, $T$ ) and we define in an analogous way

$$
\varphi_{\alpha}^{T}=\alpha T+(1-\alpha) E A \quad \alpha \in[0,1],
$$

can we obtain with $\varphi_{\min }^{T}$ all the results we have obtained with $\varphi_{\min }$ ? The answer is negative, as we have yet mentioned. The main result, that shows 
the equivalence between finding the $\alpha^{*}$ and applying $\varphi_{\text {min }}$, or assigning to each agent the smallest claim and distribute the remaining estate by using the Talmud solution, is no longer true, as the following example shows: ${ }^{16}$

Example 6. Consider $(E, c)=(4500,(500,2000,3500))$. Then, $T(E, c)=$ $(250,1375,2875), E A(E, c)=(1500,1500,1500), \alpha^{*}=0.8$, and we obtain: $\varphi_{\min }^{T}=(500,1400,1600)$ But, if we compute $(500,500,500)+$ $T(3000,(0,1500,3000))=(500,500,500)+(0,750,2250)=(500,1250,2750)$, which is a different result.

Note that the $\alpha_{m i n}-$ Egalitarian solution can be also understood as a kind of "Constrained Proportional" solution in the sense that it can be used to ensure a minimum amount to any agent. Suppose that a small amount $\tilde{c}<c_{1}$ must be received by each agent. ${ }^{17}$ What remains of the estate, if any, is shared proportionally among all agents. Then, given a claims problem $(E, c)$ this distribution can be obtained by using the $\alpha_{m i n}$-Egalitarian solution in the following way:

$$
\varphi(E, c):=\varphi_{\min }\left(E+\tilde{c}, c^{*}\right) \quad c^{*}=\left(c_{0}=\tilde{c}, c_{1}, \ldots, c_{n}\right)
$$

where only the last $n$-components of the $\alpha_{m i n}-$ Egalitarian solution are considered. This interpretation can be used, as we have mentioned in the Introduction, to obtain the distribution of seats in Spanish Parliament among districts. The Spanish system guarantees two seats to any district. The other seats are distributed to districts proportional to the population. Then, by applying the $\alpha_{m i n}$-Egalitarian solution with $\tilde{c}=2$ we obtain the actual distribution of seats.

\section{ACKNOWLEDGEMENTS}

Personal acknowledgements will be included.

The usual caveat applies. Financial support from Universitat Rovira i Virgili, Banco Santander and Generalitat de Catalunya

\footnotetext{
${ }^{16}$ This result is not true for any of the solutions that we have previously mentioned, apart from the Proportional and, obviously, $C E A$.

${ }^{17}$ Such situations can be found, for instance, in the distribution of a heritage; or the State's guarantee of a minimum retirement pension; fixing a minimal fishing quota, or milk quota; ...
} 
under project 2011LINE-06 and the Barcelona GSE are gratefully acknowledged.

\section{References}

Aumann, R. J., Maschler, M., 1985. Game Theoretic Analysis of a bankruptcy from the Talmud. Journal of Economic Theory 36, 195-213.

Bosmans, K., Lauwers, L., 2011. Lorenz comparisons of nine rules for the adjudication of conflicting claims. International Journal of Game Theory 40 (4), 791-807.

Curiel, J., Maschler, M., Tijs, S., 1987. Bankruptcy games. Zeitschrift für Operations Research 31, 143-159.

Dagan, N., Serrano, R., Volij, O., 1997. A non-cooperative view of consistent bankruptcy rules. Games and Economic Behavior 18, 55 - 72.

Dominguez, D., Thomson, W., 2006. A new solution to the problem of adjudicating conflicting claims. Economic Theory 28 (2), 283-307.

Dutta, B., Ray, D., 1989. A concept of egalitarianism under participation constraints. Econometrica 57, 615-635.

Grahn, S., Voorneveld, M., 2002. Population Monotonic Allocation Schemes in Bankruptcy Games. Annals of Operations Research (109), 317-329.

Herrero, C., Martínez, R., 2004. Egalitarian rules in claims problems with indivisible goods. A Discusión. w.p.

Herrero, C., Villar, A., 2002. Sustainability in bankruptcy problems. Top $10(2), 261-273$.

Moreno-Ternero, J. D., Villar, A., 2004. The Talmud rule and the securement of agents' awards. Mathematical Social Sciences 47 (2), 245-257.

O’Neill, B., 1982. A problem of rights arbitration from the Talmud. Mathematical Social Sciences 2 (4), 345-371.

Thomson, W., 2003. Axiomatic and game-theoretic analysis of bankruptcy and taxation problems: a survey. Mathematical Social Sciences 45 (3), 249-297. 
Thomson, W., 2006. How to divide when there is not enough; from the Talmud to game theory. Unpublished book manuscript.

Young, P., 1987. On dividing an amount according to individual claims or liabilities. Mathematics of Operations Research 12, 198-414.

Young, P., 1994. Equity In Theory and Practice. Princeton University Press, Princeton NJ.

\section{Appendix}

\section{A1: Proof of Proposition 1}

For each $(E, c) \in \mathcal{B}$ and given an agent $i \neq 1 \in N,\left(\varphi_{\min }(E, c)\right)_{i}=$ $\left(1-\alpha^{*}\right) \frac{E}{n}+\alpha^{*} \frac{c_{i} E}{C}=c_{1}-\alpha^{*} \frac{c_{1} E}{C}+\alpha^{*} \frac{c_{i} E}{C}==c_{i}+\left(\frac{\alpha^{*} E}{C}-1\right)\left(c_{i}-c_{1}\right) \leq c_{i}$

\section{A2: Proof of Proposition 2}

From Proposition $1, \alpha^{*}$ is the solution of the equation:

$$
\alpha P_{1}+(1-\alpha) \frac{E}{n}=c_{1},
$$

that implies

$$
\alpha=\frac{\frac{E}{n}-c_{1}}{\left(\frac{1}{n}-\frac{c_{1}}{C}\right) E}=\frac{C\left(E-n c_{1}\right)}{E\left(C-n c_{1}\right)} \quad C=\sum_{i=1}^{n} c_{i} .
$$

By observing that denominator is always positive, it is immediate to obtain that this fraction is less or equal than 1 . On the other hand, it is negative whenever $\frac{E}{n} \leq c_{1}$ and, in this case, $\alpha^{*}=0$. 


\section{A3: Proof of Proposition 3}

Given a claims problem $(E, c) \in \mathcal{B}$, it is clear that whenever $c_{1} \geq E / n$ then $\alpha^{*}(E, c)=0$ and $\varphi_{\min }(E, c)=C E A(E, c)=E / n$.

Suppose now that $c_{1}<E / n$. Then, for each $i \in N$, see Proposition 2,

$$
\begin{gathered}
\left(\varphi_{\min }(E, c)\right)_{i}=\alpha^{*} P_{i}(E, c)+\left(1-\alpha^{*}\right) E A_{i}(E, c)= \\
=\frac{C\left(E-n c_{1}\right)}{E\left(C-n c_{1}\right)} \frac{E c_{i}}{\sum_{j=1}^{n} c_{j}}+\left(1-\frac{C\left(E-n c_{1}\right)}{E\left(C-n c_{1}\right)}\right) \frac{E}{n}= \\
=\frac{E-n c_{1}}{C-n c_{1}} c_{i}+\frac{c_{1}(C-E)}{C-n c_{1}}= \\
=c_{1}+\left(E-n c_{1}\right) \frac{c_{i}-c_{1}}{C-n c_{1}}=c_{1}+P_{i}\left(E-n c_{1}, c-\mathbf{c}^{1}\right) .
\end{gathered}
$$

\section{A4: Proof of Proposition 4}

In order to check this result, note that for each $(E, c) \in \mathcal{B}$, if $c_{1} \geq \frac{E}{n}$, then the $\varphi_{\min }$ distributes the estate as the $E A$ solution, which satisfies all properties. Otherwise,

$$
\varphi_{\min }(E, c)=\mathbf{c}^{1}+P\left(E-n c_{1}, c-\mathbf{c}^{1}\right) .
$$

That is, each agent receives the smallest claim $c_{1}$ and the remaining estate $E_{1}=E-n c_{1}$ is distributed in a proportional way among the other agents. Then, Order Preservation is obvious. With respect to Resource monotonicity the only unclear case is whenever

$$
c_{1}<\frac{E^{\prime}}{n} \quad \text { and } \quad c_{1} \geq \frac{E}{n} .
$$

Then,

$$
\varphi_{\min }(E, c)=\frac{E}{n}, \quad \varphi_{\min }\left(E^{\prime}, c\right)=\mathbf{c}^{1}+P\left(E_{1}^{\prime}, c-\mathbf{c}^{1}\right)
$$

and the property is fulfilled. A similar reasoning can be made with Supermodularity and Composition down. Regarding to Composition up the only 
unclear case is whenever $c_{1}>\frac{E^{\prime}}{n}$ and $c_{1} \leq \frac{E}{n}$. But, in this case, $\varphi_{\min }\left(E^{\prime}, c\right)=$ $\varphi_{\min }\left(E^{\prime}, \varphi_{\min }(E, c)\right)=\frac{E}{n}$, and the property is fulfilled.

Reasonable lower bounds on awards is satisfied, since

$$
\left(\varphi_{\min }(E, c)\right)_{i} \geq \min \left\{\frac{E}{n}, c_{1}+P_{i}\left(E_{1}, c-c^{1}\right)\right\} \geq \frac{\min \left\{c_{i}, E\right\}}{n} .
$$

Finally, in order to prove that our solution fulfills Order preservation under claims variations consider two claims problems $(E, c),\left(E, c^{\prime}\right) \in \mathcal{B}$, such that $c^{\prime}=\left(c_{k}^{\prime}, c_{-k}\right), c_{k}^{\prime}<c_{k}$, and consider $i, j \in N \backslash k$ with $c_{i} \leq c_{j}$. We have the following possibilities:

(1.) If $c_{1} \geq c_{1}^{\prime} \geq \frac{E}{n}$, then the $\alpha_{\text {min }}$ distributes the estate as the $C E A$ solution, which satisfies Order preservation under claims truncation.

(2.) If $c_{1} \geq \frac{E}{n}>c_{1}^{\prime}$, then $k=1$ and

$$
\left(\varphi_{\min }\right)_{i}(E, c)=\frac{E}{n} \quad\left(\varphi_{\min }\right)_{i}\left(E, c^{\prime}\right)=c_{1}^{\prime}+\frac{E-n c_{1}^{\prime}}{\sum_{i \in N \backslash 1}\left(c_{i}-c_{1}^{\prime}\right)}\left(c_{i}-c_{1}^{\prime}\right) .
$$

So, for each pair $i, j \in N \backslash 1$ with $c_{i} \leq c_{j}$,

$$
\begin{gathered}
{\left[\left(\varphi_{\text {min }}\right)_{i}\left(E, c^{\prime}\right)-\left(\varphi_{\text {min }}\right)_{i}(E, c) \leq\left(\varphi_{\text {min }}\right)_{j}\left(E, c^{\prime}\right)-\left(\varphi_{\text {min }}\right)_{j}(E, c)\right] \Leftrightarrow} \\
\Leftrightarrow\left[c_{1}^{\prime}+\frac{E-n c_{1}^{\prime}}{\sum_{i \in N \backslash 1}\left(c_{i}-c_{1}^{\prime}\right)}\left(c_{i}-c_{1}^{\prime}\right)-\frac{E}{n} \leq c_{1}^{\prime}+\frac{E-n c_{1}^{\prime}}{\sum_{i \in N \backslash 1}\left(c_{j}-c_{1}^{\prime}\right)}\left(c_{j}-c_{1}^{\prime}\right)-\frac{E}{n}\right] \Leftrightarrow \\
\Leftrightarrow\left[c_{i}-c_{1}^{\prime} \leq c_{j}-c_{1}^{\prime}\right] \Leftrightarrow c_{i} \leq c_{j} .
\end{gathered}
$$

(3.) If $c_{1} \leq \frac{E}{n}$, then

$$
\left(\varphi_{\min }\right)_{i}(E, c)=c_{1}+\frac{E-n c_{1}}{\sum_{i \in N \backslash 1}\left(c_{i}-c_{1}\right)}\left(c_{i}-c_{1}\right)
$$

(3.1.) If $k=1$, for each pair $i, j \in N \backslash 1$ with $c_{i} \leq c_{j}$,

$$
\begin{gathered}
{\left[\left(\varphi_{\min }\right)_{i}\left(E, c^{\prime}\right)-\left(\varphi_{\min }\right)_{i}(E, c) \leq\left(\varphi_{\min }\right)_{j}\left(E, c^{\prime}\right)-\left(\varphi_{\min }\right)_{j}(E, c)\right] \Leftrightarrow} \\
\Leftrightarrow\left[c_{1}^{\prime}+\frac{E-n c_{1}^{\prime}}{\sum_{i \in N \backslash 1}\left(c_{i}-c_{1}^{\prime}\right)}\left(c_{i}-c_{1}^{\prime}\right)-c_{1}-\frac{E-n c_{1}}{\sum_{i \in N \backslash 1}\left(c_{i}-c_{1}\right)}\left(c_{i}-c_{1}\right) \leq\right.
\end{gathered}
$$




$$
\begin{gathered}
\left.\leq c_{1}^{\prime}+\frac{E-n c_{1}^{\prime}}{\sum_{i \in N \backslash 1}\left(c_{j}-c_{1}^{\prime}\right)}\left(c_{j}-c_{1}^{\prime}\right)-c_{1}-\frac{E-n c_{1}}{\sum_{i \in N \backslash 1}\left(c_{j}-c_{1}\right)}\left(c_{j}-c_{1}\right)\right] \Leftrightarrow \\
\Leftrightarrow\left[\frac{E-n c_{1}^{\prime}}{\sum_{i \in N \backslash 1}\left(c_{i}-c_{1}^{\prime}\right)}\left(c_{i}-c_{1}^{\prime}\right)-\frac{E-n c_{1}}{\sum_{i \in N \backslash 1}\left(c_{i}-c_{1}\right)}\left(c_{i}-c_{1}\right) \leq\right. \\
\Leftrightarrow\left[\frac{E-n c_{1}^{\prime}}{\sum_{i \in N \backslash 1}\left(c_{j}-c_{1}^{\prime}\right)}\left(c_{j}-c_{1}^{\prime}\right)-\frac{E-n c_{1}}{\sum_{i \in N \backslash 1}\left(c_{j}-c_{1}\right)}\left(c_{j}-c_{1}\right)\right] \Leftrightarrow \\
{\left[\frac{E-n c_{1}}{\sum_{i \in N \backslash 1}\left(c_{j}-c_{1}\right)}\left(c_{j}-c_{i}\right) \leq \frac{E-n c_{1}^{\prime}}{\sum_{i \in N \backslash 1}\left(c_{j}-c_{1}^{\prime}\right)}\left(c_{j}-c_{i}\right)\right] \Leftrightarrow c_{1}^{\prime} \leq c_{1} .}
\end{gathered}
$$

(3.2.) If $k \neq 1$, then

$$
\begin{aligned}
& \left(\varphi_{\text {min }}\right)_{i}(E, c)=c_{1}+\frac{E-n c_{1}}{\sum_{i \in N \backslash 1}\left(c_{i}-c_{1}\right)}\left(c_{i}-c_{1}\right) \\
& \left(\varphi_{\text {min }}\right)_{j}(E, c)=c_{1}+\frac{E-n c_{1}}{\sum_{i \in N \backslash 1}\left(c_{i}-c_{1}\right)}\left(c_{j}-c_{1}\right),
\end{aligned}
$$

and the property is fulfilled.

Clearly, by the way we have defined our consistent extension (see Remark 4), the $\alpha_{m i n}-$ Egalitarian solution fulfills Limited consistency.

\section{A5: Proof of Proposition 6}

Consider a claims problem $(E, c) \in \mathcal{B}$.

(1.) If $c_{1} \leq \frac{E}{n}$, and we name $\left(x_{1}, x_{2}, \ldots, x_{n}\right)=\varphi_{\min }(E, c)$

$$
x_{i}=c_{1}+\frac{c_{i}-c_{1}}{C-c_{1}}\left(E-n c_{1}\right) ; \quad C=\sum_{i=1}^{n} c_{i}
$$




$$
\begin{aligned}
& E^{\prime}=E-x_{n}=(n-1) c_{1}+\left(E-n c_{1}\right)-\frac{c_{n}-c_{1}}{C-n c_{1}}\left(E-n c_{1}\right) \\
& c^{\prime}=\left(c_{1}, c_{2}, \ldots, c_{n}-1\right) ; \quad C^{\prime}=C-c_{n} ; \quad c_{1} \leq \frac{E^{\prime}}{n-1} .
\end{aligned}
$$

Then,

$$
\left(\varphi_{\min }\right)_{i}\left(E^{\prime}, c^{\prime}\right)=c_{1}+\frac{c_{i}-c_{1}}{C^{\prime}-c_{1}}\left(E^{\prime}-(n-1) c_{1}\right), \quad i=1,2, \ldots, n-1,
$$

which coincides with $x_{i}$.

(2.) If $c_{1}>\frac{E}{n}$, then $\varphi_{\min }(E, c)=E A(E, c)=\frac{E}{n}$ and the property is fulfilled.

\section{A6: Proof of Proposition 7}

a) For each $(E, c) \in \mathcal{B}$ and each $i \in N$, it follows from Bosmans and Lauwers (2011) that $\varphi_{\text {min }}$ Lorenz dominates $C E L$. In order to prove that it also dominates the proportional solution $P$, some notation will help. Given a vector $\mathbf{x}=\left(x_{1}, x_{2}, \ldots, x_{n}\right)$ we define the partial sums vector:

$$
\mathbf{z}_{x}=\left(x_{1}, x_{1}+x_{2}, \ldots, x_{1}+x_{2}+\ldots+x_{n}\right)
$$

Then, $\mathbf{x} \succ_{L} \mathbf{y} \quad \Leftrightarrow \quad \mathbf{x} \neq \mathbf{y}$ and $\left(\mathbf{z}_{x}\right)_{i} \geq\left(\mathbf{z}_{y}\right)_{i}$. Now denote:

$$
\mathbf{x}=E A(E, c) \quad \mathbf{y}=P(E, c)
$$

We know that $\mathbf{x} \succ_{L} \mathbf{y}$, so $\left(\mathbf{z}_{x}\right)_{i} \geq\left(\mathbf{z}_{y}\right)_{i}$. For each $\alpha \in[0,1]$,

$$
\alpha\left(\mathbf{z}_{y}\right)_{i}+(1-\alpha)\left(\mathbf{z}_{x}\right)_{i} \geq \alpha\left(\mathbf{z}_{y}\right)_{i}+(1-\alpha)\left(\mathbf{z}_{y}\right)_{i}=\left(\mathbf{z}_{y}\right)_{i} .
$$

We conclude that $\left(\mathbf{z}_{\varphi_{\text {min }}}(E, c)\right)_{i} \geq\left(\mathbf{z}_{y}\right)_{i}$ and then $\varphi_{\min }(E, c) \succ_{L} P(E, c)$. 